\title{
A rational drug designing: What bioinformatics approach tells about the wisdom of practicing traditional medicines for screening the potential of Ayurvedic and natural compounds for their inhibitory effect against COVID-19 Spike, Indian strain Spike, Papain-like protease and Main Protease protein
}

\author{
Prashant Ranjan \\ Banars Hindu University \\ Bhagyalaxmi Mohapatra \\ Banars Hindu University \\ Parimal Das ( $\sim$ parimal@bhu.ac.in ) \\ Banaras Hindu University
}

\section{Research Article}

Keywords: Natural compounds, Virtual Screening, Molecular Docking, Drug Repositioning, Anti-Viral drugs, COVID-19, Molecular network, Indian strain Spike

Posted Date: May 20th, 2020

DOI: https://doi.org/10.21203/rs.3.rs-30366/v1

License: (c) (1) This work is licensed under a Creative Commons Attribution 4.0 International License. Read Full License 


\section{Abstract}

The new outbreak of Coronavirus disease (COVID-19) has been entitled as a pandemic by W.H.O. It spreads to almost 211 countries due to its contagious nature. There is an urgent need for developing specific therapeutic agents against COVID19. We have performed virtual screening of 52 ligands; most of which are essential components of traditional Ayurvedic medicine as well as natural compounds and three were standard repurposing drugs against COVID-19 Spike, Indian strain Spike (IS-Spike), PL-Pro and M-Pro to find potential inhibitor effects. Based on the docking results, it is estimated that compounds have a better binding affinity with M-Pro and PL-Pro than Spike as well as IS-Spike so it can be beneficial as therapeutics against COVID-19. We also conclude that the binding affinity of ligands with IS-Spike gets low as compared to Spike so the inhibitory potential of drugs may get weak. Based on the calculation of average binding energy (B.E) with the three targets, Spike, PL-Pro, and M-Pro, we found10 best ligands viz. (1) Punicafolin (2) Emblicanin A (3) Punigluconin (4) Lopinavir (5) Kuwanon X (6) Rutin (7) Lithospermic Acid (8) Phyllanemblinin A (9) Amarogentin and (10) Amaroswerin for inhibition. These ligands may act as potential inhibitors against COVID-19 druggable tri-targets. Network analysis revealed that four ligands out of 10 leading compounds are common in all four different networks (Spike, IS-Spike, PL-Pro, and MPro) which come under Phyllanthus emblica. Notably, a compound of Azadirachta indica out of 4 and a single compound of Swertia chirata was found common in all networks. Additionally, a standard drug Lopinavir and a compound of Salvia miltiorrhiza are frequently found in all networks. In principle, it appears plausible that the compounds which are common in the entire network should have more inhibitory potential against COVID19 due to the better binding potential among all targets, thus providing better candidacy for drug development.

\section{Introduction}

Another episode of pneumonia brought about by novel corona virus disease (COVID-19) of obscure aetiology, which first time rose in Wuhan, China toward the end of December 2019 with exceptionally infectious virus (human to human) [1]. This virus has affected numerous peoples of China and now it spreads around 211 countries in very short duration and it becomes pandemic. COVID-19 has been announced as a general wellbeing crisis of worldwide worry by the World Health Organization [2]. Phylogenetic evolutionary analysis revealed that the COVID-19 have 79\% nucleotide homology with SARSCoV, 96\% nucleotide homology with bats SARS-CoV and 50\% identity with MERS- CoV [3], [4].

Although specific vaccines and antiviral operators are the highly effective strategies to forestall and treat viral disease, no viable medications are available till datethat focus on the COVID-19 infections [5]. Currently Hydroxychloroquine and azithromycin used as a treatment of COVID- 19 [6]. Some antiviral drugs like Favinapir, Ritoavir, Oseltamivir, Lopinavir, Ganciclovir and Remdesivir are clinically tested against COVID-19 infection [7]. According to the World Health Organisation, there are eight corona virus vaccines candidates those enter for human trials and 100 are in pre-clinical stage.

Development of this therapeutics or vaccines may require months or years. Mean while an immediate therapeutics or control mechanism should therefore be actively searched.

In several countries like in India those have insufficient modern medical resources have rich resources of medical plants and those have been continued in practice in the treatment of different diseases. In fact, employments of conventional drugs are primarily supported in the majority of the world's population [8].One of the significant motivations to separate and procedure conventional restorative compounds from plants are in rising practice because of their least side effect than other allopathic prescriptions [9]. Ayurveda, the conventional Indian restorative framework remains the very ancient yet living customs with the sound philosophical and trial premise.The utilization of herbal treatment is equally significant in this Indian conventional arrangement of medication by the self-healing properties [10]. The adequacy of herbal treatment to control infectious illness was shown at some stage in the 2003 Severe Acute Respiratory Syndrome (SARS) flare-up [11]. 
The COVID-19 encodes several proteins, some of which are essential to viral ingress and replication. Papain-like protease (PL-Pro), Main protease (M-Pro) and Spike protein are the best- contemplated proteins among them and make appealing focuses for the sedate turn of events. Coronavirus PL-Pro is a deubiquitinating protein that can reduce host anti-viral response by capturing the ubiquitin (Ub) framework. SARS PL-Pro divides ISG15 into two-domains, Ub-like protein, and Lys48-connected poly Ub chains, discharging di Ub Lys48 items [12], [13]. SARS-3CLpro or main protease (M-Pro) is a cysteine protease which is responsible for maturation of the polyproteins and essential to the lifecycle [14]. Spike (S) glycoprotein ties to the cell layer protein angiotensin-converting enzyme 2 (ACE2) to enter human cells. During the infectious condition, the S protein is severed into subunits, S1, and S2. However, S1 contains the receptor- binding domain (RBD) which permits corona viruses to legitimately tie to the peptidase domain (PD) of ACE2. In addition to S2 likely assumes a job in membrane layer combination [15]. Mutational analysis of the COVID-19 genomes from different geographical location mainly India, Italy, USA, Nepal and Wuhan revealed a unique mutation found in spike surface glycoprotein (A930V) (24351C>T) in the Indian COVID-19 and it absent in other strains of different geographical origins [16].

Through Computational molecular virtual screening of small molecules from natural compounds, have been confirmed to directly inhibit these important proteins in SARS or Middle East respiratory syndrome (MERS) coronavirus [17], [18], [19], [20], [21], [22]. Genome sequence analysis of COVID-19 revealed that the main proteins of COVID-19 have highest similarities with SARS-CoV and MERS-CoV [3].

Nucleozin is a compound, which found by arbitrary screening which actuates NP conglomeration, by repressing viral replication [23]. Several compounds from therapeutic plants have been accounted for to have antiviral bioactivities [24], [25], [26].

In the current examination, we have completed the virtual screening of 52 ligands; the vast majority of which is a basic part of numerous traditional Ayurvedic medication just as others natural compounds and three of them are standard repurposing drugs against COVID-19 M-Pro, PL-Pro, Spike and IS-Spike to locate the potential inhibitors of its catalytic domain. In addition, three standard repurposing drugs utilizing nowadays against COVID19 infection strain proteins were likewise utilized for correlation through atomic docking. Furthermore we also compared the binding affinity of Spike and ISSpike with ligands and evaluate the drug effectiveness and determination of their interaction pattern between them. The discoveries of this examination will prescribe different specialists with chances to distinguish the exact medication to battle COVID- 19.

\section{Materials And Methods}

\section{Experimental investigation}

With the purpose of analyzing the nature of molecular interaction of 52 ligands with their targets, the fundamental experimental approach involved retrieval of the accessible 3D structures of ligands and its targets along with the prediction of the unavailable structures of COVID-19 IS- Spike and PL-Pro. Docking analyses were carried out by HEX 8.0 and PatchDock Server. In addition, the docked complexes were visualized by Discovery Studio 3.5. A flowchart of the experimental design is delineated in Fig.1. The flow diagram shows the experimental approach and tools used to retrieve the 3D structure of targets, docking investigation, and representation of docked complexes.

\section{Retrieval of ligands and target structures}

The 3D structure of ligands was obtained from the PubChem Compound database, followed by its conversion from the SDF to PDB format. The molecular structures of Weddolactone and Desmethylweddolactone were modeled using ACD/Chem-Sketch because it was not available in PubChem database. Open Babel [27] was used for file format 
conversion (.mol into pdb) and optimization of all ligands structure was done using Discovery Studio 3.5

(http://accelrys.com/products/collaborative-science/biovia-discovery-studio/visualization- download.php). The 3D structures of the COVID-19 Spike and M-Pro were retrieved from Protein Data Bank (PDB) (https://www.rcsb.org/).

\section{Generation of target structures of COVID-19 PL-Pro and Indian Strain Spike}

As the structures of COVID-19 PL-Pro and IS-Spike was unavailable in the Protein Data Bank, approaches of $A b$ initio predictions of PL-Pro using I-TASSER [28] and Homology modeling was used for predicting the 3D structure of IS-Spike using Swiss model. Templates used for prediction of IS-Spike structure were 6vsb (PDB ID). The quality of the derived structures was further validated by PDBsum server following method described by Yadav et al., 2017 [29]. The 3D structures thus generated were visualized by Discovery Studio 3.5 .

\section{Energy minimization}

All targeted structure whether downloaded from protein data bank or generated from I-TASSER and Swiss model are additionally refined by molecular dynamic programme YASARA (Yet Another Scientific Artificial Reality Application, http://www.yasara.com) [30]. YASARA server calculates the initial and final energy of COVID-19 protein, which is based on NOVA (Nucleotide Optimization in VAcuo).

\section{Docking analysis}

Docking of Ligands with various selected targets was carried out by Hex8.0 and PatchDock Server [29] (default parameter RMSD esteem 4.0 and complex type protein-small ligand) trailed by visualization of the docked complexes through Discovery Studio 3.5. Docking investigation was based on geometric shape complementarity score (GSC score), which was determined in PatchDock Server, and binding energy evaluated using Hex8.0. By default parameter like grid dimension (0.6), receptor range (180), ligand range (180), twist range (360) distance range (40), scan step (0.8) was used for the interaction study, assuming ligand is rigid. Docking investigation of Hex depends on spherical polar Fourier (SPF) calculation [31]. The findings of the results are based on the docking energy value and the interaction at the binding sites. The more negative value indicates minimum e-value represents more binding affinity hence more steady the complex. In accordance with energy funnel theory less energy depicts very stable conformation. Hence, more energy would be required to break the complex that means elevated dissociation energy. The optimum clusters were selected on the basis of e-value (Binding energy) in Hex 8.0.

\section{Molecular Interaction network}

The molecular interaction network of top ten interacting ligands with their selected targets was created in Cytoscape 2.8.3 software [32].

\section{Results And Discussion}

\section{Ligands preparation and Target selection}

In order to carry out the docking analysis, in the beginning the 3D structures of 49 ligands (natural compounds) out of 52 (Table1) were retrieved from PubChem Compound database and three ligands were modeled using ACD/Chem-Sketch. 
Many natural compounds reported to have biologically confirmed anti-SARS and anti-MERS coronavirus activity were identified [33], [34], [16], [17], [18], [19]. We have selected 21 medicinal plants that have anti-viral property (Table 1 \& 4) and their compounds have high probability of directly inhibiting the COVID-19, perhaps providing immediate help in the treatment of the diseases which cause pneumonia.

The prospective targets were selected on the basis of functional categories of molecules: (1) The deubiquitinating enzyme PL-Pro that can reduce host anti-viral response [12], [13] (2) 3CLpro or main protease is important to the virus lifecycle [35]. (3) Spike binds to the cell membrane protein to enter human cells [14].These three targets provide a great opportunity to identify potential drug candidates for the treatment. COVID-19 3CL-Pro/M-Pro (PDB ID: 6LU7) [36] and Spike (PDB ID: 6vsb) [37] structures were retrieved from PDB data bank, whereas the structure of PL-Pro and IS-Spike was determined by $A b$-initio modeling and homology modeling approach as the same was unavailable at PDB data bank. Energy minimization of all targeted 3D structure was done by YASARA server. Initial energy of the Spike, IS-Spike, M-Pro and PL-Pro protein were $-135274.6,-378170.5,-126585.8$ and $12117262.4 \mathrm{~kJ} / \mathrm{mol}$ respectively while the end energy of the model is -146567.6 , $-534242.4,-166967.6$ and $-848519.5 \mathrm{~kJ} / \mathrm{mol}$ respectively. Energy minimization removes severe steric clashes between two atoms with a distance [30]. The 3D-Structure of COVID-19 retrieved as well as predicted model was further validated with PDBsum. Ramachandran plot analysis of the Spike, IS-Spike, M-Pro and PL-Pro model showed 83.7\%, 86.2\%, 91.7\% and $74.7 \%$ residues come under favored region. The two model which are close to the requisite percentage of 90 for validating model which is near to good model, one cross to 90 i.e. good model, and one below 80 which come under average model. (Fig.2.). Favoured region residues varied between Spike and IS-Spike due to a change of A930V (24351C>T) in IS-Spike COVID-19 (Indian mutant Spike), which revealed that IS-Spike is more stable than general Spike protein. Favoured regions which showed in Ramachandran plot are energetically and sterically stable conformations of residues characterized by values of torsion angles $\psi$ and $\phi$. Mutant position in Ramachandran Plot for Predicting Protein Stability of Surface Mutations [38].

\section{Docking studies}

With the aim of findinga potential candidate for treating COVID-19, molecular docking studies were performed out the virtual screening of 52 ligands. Most of the molecules are active against different viral diseases viz. HIV, Influenza, Herpes, West Nile virus, and Dengue virus. The list of ligands tested for docking study is depicted in Table 1.

Protein-ligand interactions were also decoded with respect to nature of interacting amino acid residues and association of $\mathrm{H}$-bonding. Binding of ligands to the target also indicates to the possibility that the ligand may be capable of ushering functional modulations in the target molecule [39], [40]. Most of the interacting amino acidic residues along with hydrogen bonding interactions shown by PatchDock analysis and further visualized by Discovery studio 3.5 (Fig.4- 7). The estimation of binding energy evaluation done by Hex8 to provide precious information that can be used to scrutinize the results of approving studies, for instance, in vitro, in vivo and clinical studies.

All 52 molecules were docked against the target enzyme COVID-19 and ranked based on their dock scores (Binding energy) and were depicted in Table3. In this table, we have mentioned the B.E of each molecule with Spike, IS-Spike, PL-Pro and MPro. The column average (avg.) B.E with Spike is avg. of Spike, PL-Pro and M-Pro. Similarly, the column avg. B.E with ISSpike is avg. of IS-Spike, PL-Pro and M-Pro. Ranking is based on the avg. value of data, in which Rank1 indicates highest avg. binding energy with particular molecule and Rank 2 indicates lesser binding energy as compared to Ranked 1 molecule and so on. We have selected top 10 ligands which have high binding affinity to their targets i.e. Spike, IS-Spike, PL-Pro and M-Pro on the basis of binding energy (calculated by docking) and were depicted in Table2.The 10 leading ligands have high binding affinity for inhibition of Spike protein was Punicafolin (Phyllanthus emblica), Emblicanin A (Phyllanthus emblica), Lopinavir (Anti-HIV drugs), Punigluconin (Phyllanthus emblica), Kuwanon X (Morus alba), Rutin (Azadirachta indica), Phyllanemblinin A (Phyllanthus emblica), Lithospermic Acid (Salvia miltiorrhiza), Amaroswerin 
(Swertia chirata) and Heptacosanol (Eclipta prostrata).The standard drugs like Lopinavir, Hydroxychloroquinine and Ribavirin, which interact with Spike plus it come under 3, 15 and 21 rank out of 52 . First 10 ligands have high binding affinity for inhibition of IS-Spike protein was Punigluconin (Phyllanthus emblica), Emblicanin A (Phyllanthus emblica), Rutin (Azadirachta indica), Lopinavir (Anti HIV drugs), Punicafolin (Phyllanthus emblica), Amarogentin (Swertia chirata), Azadirachtin (Azadirachta indica), Amaroswerin (Anti HIV drugs), Phyllanemblinin A (Phyllanthus emblica) and Lithospermic Acid (Salvia miltiorrhiza). The standard drugs such as Lopinavir, Hydroxychloroquinine and Ribavirin, which interact with Spike as well as they ranked on 4, 20 and 41. The 10 major ligands have high binding affinity for inhibition of PL-Pro protein was Punicafolin (Phyllanthus emblica), Emblicanin A (Phyllanthus emblica), Punigluconin (Phyllanthus emblica), Lopinavir (Anti HIV drugs), Phyllanemblinin A (Phyllanthus emblica), Amarogentin (Swertia chirata), Lithospermic Acid (Salvia miltiorrhiza), Azadirachtin (Azadirachta indica), Rutin (Azadirachta indica) and Amaroswerin (Swertia chirata). The standard drugs viz. Lopinavir, Hydroxychloroquinine and Ribavirin, which interact with PL-Pro and they ranked on 4, 20 and 41. The top 10 ligands have high binding affinity for inhibition of M-Pro protein was Punicafolin (Phyllanthus emblica), Emblicanin A (Phyllanthus emblica), Punigluconin (Phyllanthus emblica), Lopinavir (Anti HIV drugs), Kuwanon x (Morus alba), Rutin (Azadirachta indica), Lithospermic Acid (Salvia miltiorrhiza), Amaroswerin (Swertia chirata), Phyllanemblinin A (Phyllanthus emblica) and Amarogentin (Swertia chirata).The standard drugs i.e. Lopinavir, Hydroxychloroquinine and Ribavirin, which interact with M-Pro and it fall under rank 4, 24 and 43. The binding affinity of ligands with M-Pro and PL-Pro is better than Spike and IS-Spike so M-Pro and PL-Pro is better inhibitory target protein for inhibitory drugs. After comparative analysis of Spike and IS-Spike, we conclude that binding affinity of ligands with ISSpike get low as compared to Spike so inhibitory potential of drugs may fragile (Table2 and 3).

Based on the avg. B.E with Spike, PL-Pro and M-Pro (Table3), we preferred best 10 ligands \{Punicafolin (Phyllanthus emblica), Emblicanin A (Phyllanthus emblica), Punigluconin (Phyllanthus emblica), Lopinavir (Anti HIV drugs), Kuwanon X (Morus alba), Rutin (Azadirachta indica), Lithospermic Acid (Salvia miltiorrhiza), Phyllanemblinin A (Phyllanthus emblica), Amarogentin (Swertia chirata) and Amaroswerin (Swertia chirata)\} for inhibition of all three targets (Spike, PL-Pro and MPro). On the basis of avg. B.E of IS-Spike, PL-Pro and M-Pro (Table3.), we favoured best 10 ligands \{Punicafolin (Phyllanthus emblica), Emblicanin A (Phyllanthus emblica), Punigluconin (Phyllanthus emblica), Lopinavir (Anti HIV drugs) Rutin (Azadirachta indica), Amarogentin (Swertia chirata) and Amaroswerin (Swertia chirata), Phyllanemblinin A (Phyllanthus emblica), Lithospermic Acid (Salvia miltiorrhiza) and Kuwanon x (Morus alba) for inhibition of three targets (IS-Spike, PL-Pro and M-Pro). Based on the avg. B.E of Spike, PL-Pro and M-Pro as well as IS-Spike, PL-Pro and M-Pro, the standard drugs Lopinavir come under $4^{\text {th }}$ rank, Hydroxychloroquine on $23^{\text {rd }}$ rank and Ribavirin on $40^{\text {th }}$ rank, out of 52 ligands.

In Table4, 49 compounds have been taken from 21 different medicinal plants and rests of three were standard drugs. Avg. value is calculated on the basis of each medicinal plant's compound i.e.

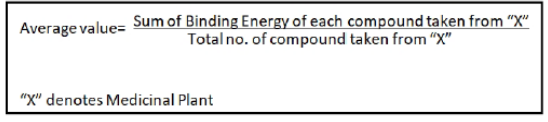

We have ranked 21 medicinal plants (possess 49 ligands) along with standard drugs (3 drugs) (Table4.) based on Avg. B.E $(\mathrm{KJ} / \mathrm{mol})$ with Spike, PL-Pro and M-Pro, and similarly ranked 21 medicinal plants (possess 49 ligands) along with standard drugs (3 drugs) (Table4) on the basis of Avg. B.E (KJ/mol) with Indian strain Spike, PL-Pro and M-Pro. From table 4 it is very clear that Malberry (Morus alba) is the most effective plant as compared to the other plants and standard drugs. While Amla (Phyllanthus emblica) is the second most effective medicinal plant. But in case of IS-Spike Amla (Phyllanthus emblica) is the most effective medicinal plant when compared with others.

There are four ligands out of top 10 whichare common in all four different networks and those come from Phyllanthus emblica (Amla) plus minimum one compound (Rutin) of Azadirachta indica ( Neem) out of 4 and a compound ( 
Amaroswerin ) of Swertia chirata, which is common in all networks. Moreover, a standard drug Lopinavir and a compound (Lithospermic Acid) of Salvia miltiorrhiza are common in all networks. Therefore, it looks logical that the compounds which are common in the entire network should have more inhibitory potential against COVID19 due to the better binding potential among all three target proteins including IS-Spike as displayed in Fig.3.

Table2. Comparative analysis of top 10 selected valuable ligands interacted with COVID-19 Spike, Indian mutant Spike, PL-Pro and M-Pro.

\begin{tabular}{|c|c|c|c|c|c|c|c|c|c|c|c|c|}
\hline Top ran king & $\begin{array}{l}\text { Molecular } \\
\text { name }\end{array}$ & $\begin{array}{c}\text { GCC } \\
\text { Spike }\end{array}$ & $\begin{array}{c}\text { B.E } \\
\text { (KJ/mol } \\
\text { ) } \\
\text { of Spike }\end{array}$ & $\begin{array}{l}\text { Molecular } \\
\text { name }\end{array}$ & $\begin{array}{l}\text { GCC } \\
\text { India } \\
\text { mut Spike }\end{array}$ & $\begin{array}{c}\text { B.E } \\
(\mathrm{KJ} / \mathrm{mol} \\
) \\
\text { of Indian } \\
\text { mutant }\end{array}$ & $\begin{array}{l}\text { Molecular } \\
\text { name }\end{array}$ & $\begin{array}{l}\text { GCC PL- } \\
\text { Pro }\end{array}$ & $\begin{array}{c}\text { B.E } \\
(\mathrm{KJ} / \mathrm{mol} \\
\text { ) of PL- } \\
\text { Pro }\end{array}$ & $\begin{array}{l}\text { Molecular } \\
\text { name }\end{array}$ & $\begin{array}{l}\text { GCC M- } \\
\text { Pro }\end{array}$ & $\begin{array}{c}\text { B.E } \\
(\mathrm{KJ} / \mathrm{mol} \\
\text { ) of } \mathrm{M}- \\
\text { Pro }\end{array}$ \\
\hline 1 & Punicafolin & 6628 & -293.66 & Punigluconin & 6736 & -249.01 & Punicafolin & 7990 & -452.28 & Punicafolin & 6878 & -492.34 \\
\hline 2 & Emblicanin A & 6336 & -292.51 & Emblicanin A & 6794 & -247.92 & Emblicanin A & 7056 & -446.87 & Emblicanin A & 6380 & -458 \\
\hline 3 & Lopinavir & 7258 & -280.47 & Rutin & 6354 & -240.45 & Punigluconin & 7452 & -434.4 & Punigluconin & 6492 & -456.87 \\
\hline 4 & Punigluconin & 6654 & -274.05 & Lopinavir & 7524 & -232.54 & Lopinavir & 8902 & -394.06 & Lopinavir & 7070 & -425.97 \\
\hline 5 & Kuwanon $\mathrm{x}$ & 6256 & -258 & Punicafolin & 7130 & -229.36 & $\begin{array}{l}\text { Phyllanemblinin } \\
\text { A }\end{array}$ & 6826 & -385.38 & Kuwanon $\mathrm{x}$ & 5768 & -405.1 \\
\hline 6 & Rutin & 6216 & -242.48 & Amarogentin & 6356 & -229.13 & Amarogentin & 7126 & -383.64 & Rutin & 6156 & -384.76 \\
\hline 7 & $\begin{array}{l}\text { Phyllanemblinin } \\
\text { A }\end{array}$ & 6050 & -234.85 & Azadirachtin & 6558 & -225.95 & $\begin{array}{l}\text { Lithospermic } \\
\text { Acid }\end{array}$ & 6854 & -383.13 & $\begin{array}{l}\text { Lithospermic } \\
\text { Acid }\end{array}$ & 5792 & -373.81 \\
\hline 8 & $\begin{array}{l}\text { Lithospermic } \\
\text { Acid }\end{array}$ & 5862 & -233 & Amaroswerin & 6274 & -223.9 & Azadirachtin & 6928 & -377.57 & Amaroswerin & 5894 & -368.89 \\
\hline 9 & Amaroswerin & 6036 & -230.98 & $\begin{array}{l}\text { Phyllanemblinin } \\
\text { A }\end{array}$ & 6072 & -204.88 & Rutin & 7056 & -367.75 & $\begin{array}{l}\text { Phyllanemblinin } \\
\text { A }\end{array}$ & 5744 & -367.1 \\
\hline 10 & Heptacosanol & 6270 & -228.92 & $\begin{array}{l}\text { Lithospermic } \\
\text { Acid }\end{array}$ & 6104 & -200.21 & Amaroswerin & 6858 & -365.55 & Amarogentin & 5808 & -365.34 \\
\hline
\end{tabular}

Table3. Comparative docking study results on COVID-19 enzymes. 


\begin{tabular}{|c|c|c|c|c|c|c|c|c|c|c|c|}
\hline $\begin{array}{l}\text { Sl.N } \\
\text { o. }\end{array}$ & Molecular name & $\begin{array}{c}\text { Source/Std } \\
\text { drugs }\end{array}$ & $\begin{array}{l}\text { Common } \\
\text { Name }\end{array}$ & $\begin{array}{c}\text { B.E } \\
\text { (KJ/mol) of } \\
\text { IS- Spike - } \\
\text { Ligands }\end{array}$ & $\begin{array}{l}\text { B.E } \\
\text { (KJ/mol) of } \\
\text { Spike- Ligands }\end{array}$ & $\begin{array}{c}\text { B.E } \\
(\mathrm{KJ} / \mathrm{mol}) \text { of } \\
\text { PL- Pro- } \\
\text { Ligands }\end{array}$ & $\begin{array}{l}\quad \text { B.E } \\
\text { (KJ/mol) of } \\
\text { M- } \\
\text { Pro- Ligands }\end{array}$ & $\begin{array}{l}\text { Avg. B.E } \\
\text { (KJ/mol } \\
\text { ) with } \\
\text { Spike }\end{array}$ & Rank & $\begin{array}{c}\text { Avg. } \\
\text { B.E(KJ/mol)with } \\
\text { IS- Spike }\end{array}$ & Rank \\
\hline 1 & Punicafolin & $\begin{array}{c}\text { Phyllanthus } \\
\text { emblica }\end{array}$ & Amla & -229.36 & -293.66 & -452.28 & -492.34 & -412.76 & 1 & -391.33 & 1 \\
\hline 2 & Emblicanin A & $\begin{array}{c}\text { Phyllanthus } \\
\text { emblica }\end{array}$ & Amla & -247.92 & -292.51 & -446.87 & -458 & -399.13 & 2 & -384.26 & 2 \\
\hline 3 & Punigluconin & $\begin{array}{c}\text { Phyllanthus } \\
\text { emblica }\end{array}$ & Amla & -249.01 & -274.05 & -434.4 & -456.87 & -388.44 & 3 & -380.09 & 3 \\
\hline 4 & Lopinavir & $\begin{array}{c}\text { Anti HIV } \\
\text { drugs }\end{array}$ & $\begin{array}{l}\text { Anti HIV } \\
\text { drugs }\end{array}$ & -232.54 & -280.47 & -394.06 & -425.97 & -366.83 & 4 & -350.86 & 4 \\
\hline 5 & Kuwanon x & Morus alba & Mulberry & -192.23 & -258 & -358.03 & -405.1 & -340.38 & 5 & -318.45 & 10 \\
\hline 6 & Rutin & $\begin{array}{c}\text { Azadirachta } \\
\text { indica }\end{array}$ & Neem & -240.45 & -242.48 & -367.75 & -384.76 & -331.66 & 6 & -330.99 & 5 \\
\hline 7 & $\begin{array}{r}\text { Lithospermic } \\
\text { Acid } \\
\end{array}$ & $\begin{array}{c}\text { Salvia } \\
\text { miltiorrhiza }\end{array}$ & Red sage & -200.21 & -233 & -383.13 & -373.81 & -329.98 & 7 & -319.05 & 9 \\
\hline 8 & $\begin{array}{r}\text { Phyllanemblinin } \\
\mathrm{A}\end{array}$ & $\begin{array}{c}\text { Phyllanthus } \\
\text { emblica }\end{array}$ & Amla & -204.88 & -234.85 & -385.38 & -367.1 & -329.11 & 8 & -319.12 & 8 \\
\hline 9 & Amarogentin & $\begin{array}{c}\text { Swertia } \\
\text { chirata }\end{array}$ & Chirayta & -229.13 & -221.5 & -383.64 & -365.34 & -323.49 & 9 & -326.04 & 6 \\
\hline 10 & Amaroswerin & $\begin{array}{l}\text { Swertia } \\
\text { chirata }\end{array}$ & Chirayta & -223.9 & -230.98 & -365.55 & -368.89 & -321.81 & 10 & -319.45 & 7 \\
\hline 11 & Azadirachtin & $\begin{array}{c}\text { Azadirachta } \\
\text { indica } \\
\end{array}$ & Neem & -225.95 & -224.46 & -377.57 & -342.81 & -314.95 & 11 & -315.44 & 11 \\
\hline 12 & Isoquercitrin & $\begin{array}{c}\text { Houttuynia } \\
\text { cordata }\end{array}$ & Fish mint & -186.09 & -205.32 & -326.61 & -315.76 & -282.56 & 12 & -276.15 & 13 \\
\hline 13 & Hentriacontanol & $\begin{array}{c}\text { Eclipta } \\
\text { prostrata }\end{array}$ & Bhringraj & -169.78 & -217.03 & -284.14 & -344.52 & -281.90 & 13 & -266.15 & 18 \\
\hline 14 & Nimbaflavone & $\begin{array}{c}\text { Azadirachta } \\
\text { indica }\end{array}$ & Neem & -199.83 & -212 & -321.59 & -311.62 & -281.74 & 14 & -277.68 & 12 \\
\hline 15 & Heptacosanol & $\begin{array}{c}\text { Eclipta } \\
\text { prostrata }\end{array}$ & Bhringraj & -170.01 & -228.92 & -284.14 & -326.21 & -279.76 & 15 & -260.12 & 21 \\
\hline 16 & Hyperoside & $\begin{array}{c}\text { Azadirachta } \\
\text { indica } \\
\end{array}$ & Neem & -188.14 & -192.9 & -328.47 & -309 & -276.79 & 16 & -275.20 & 14 \\
\hline 17 & Thalimonine & $\begin{array}{l}\text { Thalictrum } \\
\text { simplex } L .\end{array}$ & $\begin{array}{l}\text { Meadow } \\
\text { rue }\end{array}$ & -141.45 & -225.37 & -298.63 & -304.7 & -276.23 & 17 & -248.26 & 24 \\
\hline
\end{tabular}




\begin{tabular}{|c|c|c|c|c|c|c|c|c|c|c|c|}
\hline 18 & Baicalin & $\begin{array}{l}\text { Scutellaria } \\
\text { baicalensis }\end{array}$ & Baikal skullc ap & -192.07 & -195.01 & -325.29 & -304.64 & -274.98 & 18 & -274.00 & 16 \\
\hline 19 & Stigmasterol & Eclipta prostrata & Bhringraj & -184.59 & -184.29 & -333.34 & -301.94 & -273.19 & 19 & -273.29 & 17 \\
\hline 20 & Quercitrin & Houttuynia cordata & Fish mint & -184.69 & -170.57 & -340.69 & -296.85 & -269.37 & 20 & -274.08 & 15 \\
\hline 21 & Betulinic acid & Symplocos racemosa & Lodhra & -152.89 & -191.59 & -318.5 & -295.43 & -268.51 & 21 & -255.61 & 22 \\
\hline 22 & Curcumin & Curcuma longa & Turmeric & -182.11 & -193.64 & -290.36 & -316 & -266.67 & 22 & -262.82 & 19 \\
\hline 23 & Hydroxychloroquine & Anti Malarian drug & Anti Malarian drug & -174.59 & -212.31 & -302.02 & -284.17 & -266.17 & 23 & -253.59 & 23 \\
\hline 24 & Mangiferin & Swertia chirata & Chirayta & -186.26 & -179.78 & -306.83 & -287.63 & -258.08 & 24 & -260.24 & 20 \\
\hline 25 & Calanolide A & Calophyllum Spp & $\begin{array}{c}\text { Indian doomba oil } \\
\text { tree }\end{array}$ & -153.69 & -192.85 & -292.69 & -281.06 & -255.53 & 25 & -242.48 & 27 \\
\hline 26 & Gentiopicrin & Swertia chirata & Chirayta & -146.31 & -207.26 & -275.75 & -269.6 & -250.87 & 26 & -230.55 & 34 \\
\hline 27 & Flavonoid & Phyllanthus emblica & Amla & -157.89 & -181.39 & -301.3 & -269.49 & -250.73 & 27 & -242.89 & 25 \\
\hline 28 & Tinosoprin A & Tinospora cordifolia & Giloy & -133.77 & -162.75 & -289.3 & -279.49 & -243.85 & 28 & -234.19 & 29 \\
\hline 29 & Sweroside & Swertia chirata & Chirayta & -138.76 & -171.44 & -273.27 & -280.21 & -241.64 & 29 & -230.75 & 33 \\
\hline 30 & Piperine & Piper longum & $\begin{array}{c}\text { Indian long } \\
\text { pepper }\end{array}$ & -142.25 & -170.94 & -273.36 & -277.91 & -240.74 & 30 & -231.17 & 32 \\
\hline 31 & Piper longumine & Piper longum & $\begin{array}{c}\text { Indian long } \\
\text { pepper }\end{array}$ & -158.25 & -149.86 & -285.96 & -283.55 & -239.79 & 31 & -242.59 & 26 \\
\hline 32 & Swertiamarin & Swertia chirata & Chirayta & -150.87 & -168.53 & -274.78 & -273.88 & -239.06 & 32 & -233.18 & 30 \\
\hline 33 & Quercitin & Houttuynia cordata & Fish mint & -128.81 & -211.98 & -245.18 & -259.05 & -238.74 & 33 & -211.01 & 37 \\
\hline 34 & Kaempferol & Phyllanthus emblica & Amla & -129.74 & -205.25 & -248.38 & -251.81 & -235.15 & 34 & -209.98 & 38 \\
\hline 35 & Palmatine & Tinospora cordifolia & Giloy & -151.54 & -157.84 & -279.53 & -265.39 & -234.25 & 35 & -232.15 & 31 \\
\hline 36 & Jatorrohizine & Tinospora cordifolia & Giloy & -166.22 & -156.13 & -281.01 & -263.65 & -233.60 & 36 & -236.96 & 28 \\
\hline 37 & Berberine & Tinospora cordifolia & Giloy & -138.6 & -146.84 & -271.59 & -278.24 & -232.22 & 37 & -229.48 & 36 \\
\hline 38 & Magnoflorine & Tinospora cordifolia & Giloy & -156.05 & -160.59 & -272.87 & -260.3 & -231.25 & 38 & -229.74 & 35 \\
\hline 39 & Resveratrol & $\begin{array}{c}\text { Veratrum } \\
\text { grandiflorum }\end{array}$ & Corn lilies & -117.42 & -184.25 & -250.46 & -237.71 & -224.14 & 39 & -201.86 & 39 \\
\hline 40 & Ribavirin & Anti HIV drugs & Anti HIV drugs & -114.61 & -195.18 & -227.72 & -211.73 & -211.54 & 40 & -184.69 & 40 \\
\hline 41 & Zingerone & Zingiber officinale & Ginger & -100.47 & -185 & -235.15 & -207.5 & -209.22 & 41 & -181.04 & 43 \\
\hline 42 & $\begin{array}{c}\text { DesmethylWeddolact } \\
\text { one } \\
\end{array}$ & Eclipta prostrata & Bhringraj & -96.6 & -185.82 & -208.98 & -208.54 & -201.11 & 42 & -171.37 & 45 \\
\hline 43 & Weddolactone & Eclipta prostrata & Bhringraj & -105.33 & -174.16 & -205.3 & -213.9 & -197.79 & 43 & -174.84 & 44 \\
\hline 44 & Ajoene & Allium sativum L. & Garlic & -105.18 & -149.54 & -209.93 & -232.46 & -197.31 & 44 & -182.52 & 41 \\
\hline 45 & Harmine & Peganum harmala & Wild rue & -115.55 & -151.01 & -208.06 & -220.17 & -193.08 & 45 & -181.26 & 42 \\
\hline 46 & Bicyclogermacrene & Glechon marifolia & Mint & -109.44 & -159.85 & -190.79 & -201.21 & -183.95 & 46 & -167.15 & 46 \\
\hline 47 & Eugenol & Eugenia caryophyllus & Clove & -86.04 & -153.97 & -206.73 & -180.5 & -180.40 & 47 & -157.76 & 47 \\
\hline 48 & S-Allyl-L-cysteine & Allium sativum $L$ & Garlic & -80.79 & -170.4 & -183.93 & -177.61 & -177.31 & 48 & -147.44 & 49 \\
\hline 49 & $\begin{array}{c}\text { Diallyltrisulfide } \\
\text { (DTS) }\end{array}$ & Allium sativum L. & Garlic & -74.79 & -136.08 & -182.24 & -189.51 & -169.28 & 49 & -148.85 & 48 \\
\hline 50 & Allicin & Allium sativum $L$. & Garlic & -80.28 & -148.56 & -166.1 & -190.38 & -168.35 & 50 & -145.59 & 50 \\
\hline 51 & Homonojirimycin & Omphalea diandra & $\begin{array}{l}\text { Omphalea } \\
\text { diandra }\end{array}$ & -80.02 & -155.81 & -169.44 & -174.27 & -166.51 & 51 & -141.24 & 51 \\
\hline 52 & $\begin{array}{c}\text { Diallyl } \\
\text { disulfide(DDS) } \\
\end{array}$ & Allium sativum $L$. & Garlic & -75.21 & -126.93 & -161.81 & -181.93 & -156.89 & 52 & -139.65 & 52 \\
\hline
\end{tabular}

Table4. Comparative analysis of 21 medicinal plants along with standard drugs screened for effective medicinal plants as compared to standard drugs. 


\begin{tabular}{|c|c|c|c|c|c|c|c|}
\hline $\begin{array}{l}\text { Common } \\
\text { Name }\end{array}$ & Pharmacological function & References & $\begin{array}{l}\text { total compoun } \mathrm{d} \text { for } \\
\text { this study }\end{array}$ & $\begin{array}{l}\text { Avg. B.E } \\
\text { (KJ/mol) } \\
\text { with Spike }\end{array}$ & Rank & $\begin{array}{l}\text { Avg. B.E } \\
\text { (KJ/mol) with } \\
\text { IS- Spike }\end{array}$ & Rank \\
\hline Mulberry & Anti-viral & $([41]$ & 1 & -340.38 & 1 & -318.45 & 3 \\
\hline Amla & $\begin{array}{c}\text { Anti-viral, Antiyretic, Analgesic, } \\
\text { Antitussive, } \\
\text { Antiatherogeric } \\
\end{array}$ & [42] & 6 & -335.89 & 2 & -321.28 & 1 \\
\hline Red sage & Anti-viral & [43] & 1 & -329.98 & 3 & -319.05 & 2 \\
\hline Neem & Anti-viral & [44] & 4 & -301.28 & 4 & -299.83 & 4 \\
\hline $\begin{array}{l}\text { Anti HIV } \\
\text { drugs }\end{array}$ & Anti-viral & [45] & 2 & -289.19 & 5 & -267.77 & 6 \\
\hline Meadow rue & Anti-Influenza & [46] & 1 & -276.23 & 6 & -248.26 & 12 \\
\hline $\begin{array}{c}\text { Baikal } \\
\text { skullcap }\end{array}$ & Anti-viral & [47] & 1 & -274.98 & 7 & -274.00 & 5 \\
\hline Chirayta & $\begin{array}{c}\text { Anti-viral, Antifungal, Antiinflamatory \& } \\
\text { Anticancer }\end{array}$ & [48] & 6 & -272.49 & 8 & -266.70 & 7 \\
\hline Lodhra & Anti-viral & [49] & 1 & -268.51 & 9 & -255.61 & 9 \\
\hline Turmeric & Anti-viral & [50] & 1 & -266.67 & 10 & -262.82 & 8 \\
\hline $\begin{array}{l}\text { Anti Malarian } \\
\text { drug }\end{array}$ & Anti-viral & [51] & 1 & -266.17 & 11 & -253.59 & 11 \\
\hline Fish mint & Anti-viral \& Immuno stimulant & [52] & 3 & -263.56 & 12 & -253.75 & 10 \\
\hline $\begin{array}{l}\text { Indian } \\
\text { doomba oil } \\
\text { tree }\end{array}$ & Anti-HIV & [53] & 1 & -255.53 & 13 & -242.48 & 13 \\
\hline Bhringraj & $\begin{array}{c}\text { Anti-viral, Anti-oxidant, Antianalgesic \& } \\
\text { Antibacterial }\end{array}$ & [54] & 5 & -246.75 & 14 & -229.15 & 16 \\
\hline $\begin{array}{c}\text { Indian long } \\
\text { pepper }\end{array}$ & Anti-viral & [34] & 2 & -240.26 & 15 & -236.88 & 14 \\
\hline Giloy & Anti-HIV, Antipyretic,Anti-inflammatory & [55] & 5 & -235.03 & 16 & -232.50 & 15 \\
\hline Corn lilies & Anti-viral & [56] & 1 & -224.14 & 17 & -201.86 & 17 \\
\hline Ginger & Anti-viral & [57] & 1 & -209.22 & 18 & -181.04 & 19 \\
\hline Wild rue & Anti-viral & [58] & 1 & -193.08 & 19 & -181.26 & 18 \\
\hline Mint & Anti-viral \& Antifungal & [59] & 1 & -183.95 & 20 & -167.15 & 20 \\
\hline Clove & Anti-viral, Antifungal \& Antibacterial & [60] & 1 & -180.40 & 21 & -157.76 & 21 \\
\hline Garlic & $\begin{array}{c}\text { Antiviral, Antimicrobial, Reduce risk of } \\
\text { cardiovascular } \\
\text { diseases }\end{array}$ & [61] & 5 & -173.83 & 22 & -152.81 & 22 \\
\hline $\begin{array}{c}\text { Omphalea } \\
\text { diandra }\end{array}$ & Anti-viral & [62] & 1 & -166.51 & 23 & -141.24 & 23 \\
\hline
\end{tabular}

\section{Conclusion}

At present, COVID-19 has spreads in the entire worldwhichwas initially appeared in China (Wuhan). There is no approved medicine or vaccine presently exists to treat this disease. The main purpose of present investigation was scrutinizing several medicinal plant derived compounds that may be used to inhibit the COVID-19 contagious disease. Bioinformatics approaches can be used for rapid detection method for potential therapeutics for drug discovery. We have identified 10 leading ligands by virtual screening of 52 ligands, which have high binding affinity to their targets i.e. Spike, Indian strain Spike, PL-Pro and M-Pro on the basis of binding energy.

Based on the calculation of avg. B.E with Spike, PL-Pro and M-Pro (Table3.), we determined best 10 ligands were Punicafolin, Emblicanin A, Punigluconin, Lopinavir, Kuwanon X, Rutin, Lithospermic Acid, Phyllanemblinin A, Amarogentin and Amaroswerin for inhibition of all three targets (Spike, PL-Pro and M-Pro). Similarly, on the basis of avg. B.E of Indian strain Spike, PL- Pro and M-Pro, we identified first 10 ligands viz. Punicafolin, Emblicanin A, Punigluconin, Lopinavir, Rutin, Amarogentin, Amaroswerin, Phyllanemblinin A, Lithospermic Acid and Kuwanon X for inhibition of three targets (Indian strain Spike, PL-Pro and M-Pro).These drugs may act as potential inhibitors against COVID-19 tri-targets.

We have sorted 21 medicinal plants (have 49 ligands) along with standard drugs (3 drugs) based on avg. B.E (KJ/mol) with Spike, PL-Pro and M-Pro, and similarly ranked 21 medicinal plants along with standard drugs on the basis of Avg. B.E 
$(\mathrm{KJ} / \mathrm{mol})$ with Indian strain Spike, PL-Pro and M-Pro and conclude that Mulberry (Morus alba) is the most effective plant as compared to the other medicinal plants and standard drugs. Although Amla (Phyllanthus emblica) is the second most effective medicinal plant. However, in case of IS-Spike Amla (Phyllanthus emblica) is the most effective medicinal plant when compared with others. The predicted binding and ordering of drugs should also be useful to deduce the results of existing clinical trials that are verifying small molecule drugs for efficacy against COVID-19.

\section{Declarations}

\section{ACKNOWLEDGEMENT}

We are grateful to Centre for Genetic Disorders, Institute of Science, Banaras Hindu University, Varanasi, India for providing Internet facility.

\section{Conflict of interest: None}

\section{References}

1. A. Shereen, S. Khan, A. Kazmi, N. Bashir, \& R. Siddique, COVID-19 infection: origin, transmission, and characteristics of human corona viruses, Journal of Advanced Research., 24 (2020), pp. 91-98

2. C. Lai, T. P. Shih, W. C. Ko, H. J. Tang \& P. R. Hsueh, Severe acute respiratory syndrome coronavirus 2 (SARS-CoV-2) and coronavirus disease-2019 (COVID-19): The epidemic and the challenges, International journal of antimicrobial agents., 55 (2020), pp. 105924-105924

3. Zhou, X.L. Yang, X.G. Wang, B. Hu, L. Zhang, W. Zhang, et al., A pneumonia out break associated with a new coronavirus of probable bat origin, Nature., 579 (2020), pp. 270-273

4. L. Ren et al., Identification of a novel coronavirus causing severe pneumonia in human: a descriptive study, Chin. Med. J. (Engl.)., 133 (2020), pp. 1015-1024

5. Li \& E. De Clercq, Therapeutic options for the 2019 novel coronavirus (2019-nCoV), Nature reviews., 19 (2020), pp. 149150

6. Gautret et al., Hydroxychloroquine and azithromycin as a treatment of COVID-19: results of an open-label nonrandomized clinical trial, International journal of antimicrobial agents, Epub ahead of print., (2020) 105949

7. Cynthia, Z. Qiongqiong, L. Yingzhu, et al., Research and Development on ther-apeutic agents and vaccines for COVID19 and related human coronavirus diseases, ACS Cent. Sci., 6 (2020), pp. 315-331

8. Jalil, U.A. Ashfaq, S. Shahzadi, M.R. Javed, I. Rasul, S.U. Rehman, et al. Screening and design of anti-diabetic compounds sourced from the leavesof neem (Azadirachta indica), Bioinformation., 9 (2013), pp.1031

9. Akanksha, A.K. Srivastava, R. Maurya, Antihyperglycemic activity of compounds isolated from Indian medicinal plants, Indian J Exp Biol., 48 (2010), pp. 294-8

10. K. Semwal, S.P. Mishra, A. Chauhan, and R.B. Semwal, Adverse health effects of tobacco and role of Ayurveda in their reduction, J Med Sci., 15 (2015), pp. 139-46.

11. Chen, and T. Nakamura, Statistical evidence for the usefulness of Chinese medicinein the treatment of SARS, Phytother Res., 18 (2004), pp. 592-4

12. G. Bhoj, and Z.J. Chen, Ubiquitylation in innate and adaptive immunity, Nature., 458 (2009), pp. 430-7

13. K. Isaacson, and H.L. Ploegh, Ubiquitination, ubiquitin-like modifiers, and deubiquitination in viral infection, Cell Host Microbe., 5(2009), pp. 559-70

14. Wang, P.Yang, K. Liu, F. Guo, Y. Zhang, G. Zhang, and C. Jiang, SARS coronavirus entry into host cells through a novel clathrin- and caveolae-independent endocytic parhway, Cell Res., 18(2008), pp. 290- 301

Page $11 / 19$ 
15. Sardar, D. Satish, S. Birla, and D. Gupta, Comparative analyses of SAR- CoV2 genomes from dierent geographical locations and other coronavirus family genomes reveals unique features potentially consequential to host-virus interaction and pathogenesis, bioRxiv (2020)

16. C. Wen, Y.H. Kuo, J.T. Jan, P.H. Liang, S.Y. Wang, H.G. Liu, et al., Specific plant terpenoids and lignoids possess potent antiviral activities against severe acute respiratory syndrome coronavirus, J Med Chem., 50 (2007), pp. 4087-95

17. B. Ryu, S.J. Park, Y.M. Kim, J.Y. Lee, W.D. Seo, J.S. Chang, et al., SARS- CoV3CLpro inhibitory effects of quinonemethide triterpenes from Tripterygium regelii, Bio org Med Chem Lett., 20 (2010), pp. 1873-6

18. Y. Park, J.H. Kim, Y.M. Kim, H.J. Jeong, D.W. Kim, K.H. Park, et al., Tanshinones as selective and slow-binding inhibitors for SARS-CoV cysteine proteases, Bio org Med Chem., 20 (2012), pp. 5928-35

19. Y. Park, J.H. Kim, J.M. Kwon, H.J. Kwon, H.J. Jeong, Y.M. Kim, et al., Dieckol, a SARS-CoV 3CL(pro) inhibitor, isolated from the edible brown algae Eckloniacava. Bio org Med Chem., 21(2013), pp. 3730-7

20. H. Song, D.W. Kim, M.J. Curtis-Long, H.J. Yuk, Y. Wang, N. Zhuang, et al., Papain- like protease (PLpro) inhibitory effects of cinnamic amides from Tribulu sterrestris fruits, Biol Pharm Bull., 37 (2014), pp.1021-8

21. Y. Park, J.A. Ko, D.W. Kim, Y.M. Kim, H.J. Kwon, H.J. Jeong, et al., Chalcones isolated from Angelica keiskei inhibit cysteine proteases of SARS-CoV, J Enzyme Inhib Med Chem., 31(2016). pp. 23-30

22. Shen, J. Niu, C.Wang, B. Huang, W.Wang, N. Zhu, et al., High-throughput screening and identification of potent broadspectrum inhibitors of coronaviruses, JVirol., 93 (2019), pp. e00023-19

23. K.Semwal, S.P. Mishra, A. Chauhan, and R.B.Semwal, Adverse health effects of tobacco androle of Ayurveda in their reduction, J Med Sci., 15(2015), pp. 139-46

24. Zakaryan, E. Arabyan, A. Oo, and K. Zandi, Flavonoids: promising natural compounds against viral infections, Arch.Virol., 162 (2017), pp. 2539-2551

25. Guerriero et al., Production of plant secondary metabolites: Examples, tips and suggestions for biotechnologists, Genes (Basel)., 9 (2018), pp. 34-46

26. Zhang, K. Wu, X. Zhang, , S. Deng, and B. Peng, In silico screening of Chinese herbal medicines with the potential to directly inhibit 2019 novel coronavirus, J. Integr. Med., 18 (2020), pp. 152-158

27. Boyle NM, Banck M, James CA, et al., Open Babel: An open chemical toolbox, J Cheminform., 33 (2011), pp. 1-14

28. Zheng, Y. Li, C. X. Zhang, R. Pearce, S. M. Mortuza, Y. Zhang, Deep-learning contact-map guided protein structure prediction in CASP13, Proteins., 87 (2019), pp. 1149-1164

29. Yadav, S.K. Pandey, V.K. Singh, Y. Goel, A. Kumar, S.M. Singh, Molecular docking studies of 3-bromopyruvate and its derivatives to metabolic regulatory enzymes: implication in designing of novel anticancer therapeutic strategies. PLoS One., 12 (2017), pp. e0176403

30. Elmar, K. Guenther, V. Gert, Increasing the Precision of Comparative Models with YASARA NOVA - A Self-parameterizing Force Field, Proteins, Structure, Function, and Genetics., 47 (2002), 393-402

31. K. Jayaswal, G. C. Sahoo, P. Das, Rational Drug Designing Strategies \& Inhibitor Optimization: Anthrax Lethal Toxin Factor, Int. J. Bioautomation., 16(2012), p.p 239-250

32. Satapathy, D. Singh, P. Ranjan, D. Kumar, M. Kumar, K. V. Prabhu, and K. Mukhopadhyay, Transcriptome-wide analysis of WRKY transcription factors in wheat and their leaf rust responsive expression profiling, Molecular Genetics and Genomics, 289 (2014), pp. 1289-1306

33. Cinatl, B. Morgenstern, G. Bauer, P. Chandra, H. Rabenau, and H.W. Doerr, Glycyrrhizin, an active component of liquorice roots, and replication of SARS-associated coronavirus, Lancet., 361(2003), pp. 2045-6

34. T. Lin, W.C. Hsu, C.C. Lin, Antiviral natural products and herbal medicines, J. Tradit. Complement. Med., 4 (2014), pp. 24-3

Page $12 / 19$ 
35. Sun, H. Luo, C. Yu, T. Sun, J. Chen, S. Peng, J. Qin, J. Shen, Y. Yang, Y. Xie, K. Chen, Y. Wang, X. Shen, and H.Jiang, Molecular cloning, expression, purification, and mass spectrometric characterization of $3 \mathrm{C}$-like protease of SARS coronavirus, Protein Expression Purif., 32 (2003), pp. 302-308

36. X. Liu, B. Zhang , Z. Jin, H. Yang, Z. Rao, The crytal structure of 2019-nCoV main protease in complex with an inhibitor N3, 2020

37. Wrapp, N. Wang, K.S. Corbett, et al., Cryo-EM structure of the 2019-nCoV spike in the prefusion conformation. bioRxiv. (2020)

38. M. Gromiha, M. Oobatake, H. Kono, H. Uedaira, \& A. Sarai, Importance of mutant position in Ramachandran plot for predicting protein stability of surface mutations, Biopolymers., 64 (2002), pp.210-220

39. J. Kojetin, and T.P. Burris, Small molecule modulation of nuclear receptor con- formational dynamics: implications for function and drug discovery, Mol. Phar- macol., 83 (2013), pp. 1-8

40. Schena, R. Griss, K. Johnsson, Modulating protein activity using tethered ligands with mutually exclusive binding sites, Nat. Commun., 6 (2015), pp.7830

41. H. Lee, S. Y. Bae, M. Oh, K. H. Kim, and M. S. Chung, Antiviral effects of mulberry (Morus alba) juice and its fractions on foodborne viral surrogates, Foodborne Pathogens and Disease, 11(2014) pp. 224-229

42. S. Baliga, and J.J. Dsouza, Amla (Emblica officinalis Gaertn), a wonderberry in the treatment and prevention of cancer, Eur J Cancer Prev., 20 (2011), pp. 225-39

43. W. Wu, T.L. Pan, Y.L. Leu, Y.K. Chang, and P.J. Tai et al., Antiviral effects of Salvia miltiorrhiza (Danshen) against entero virus 71. Am J Chin Med., 35 (2007), pp.153-168

44. Tiwari, N. A. Darmani, B. Y. J. T. Yue, and D. Shukla, In vitro antiviral activity of neem (Azadirachta indica L.) bark extract against herpes simplex virus type-1 infection, Phytotherapy Research., 24 (2010), pp. 1132-1140

45. S .Khalili, H .Zhu, N.S.A Mak, Y. Yan , Y. Zhu, Novel coronavirus treatment with ribavirin: Groundwork for an evaluation concerning COVID-19, J Med Virol., (2020), pp. 1-7

46. Serkedjieva, and M.Velcheva, In Vitro Anti-Influenza Virus Activity of the Pavine Alkaloid (-)-Thalimonine Isolated from Thalictrum Simplex L. Antiviral Chemistry and Chemotherapy.,14 (2003), pp.75-80

47. Zhao, X.Y. Chen and C. Martin Scutellaria baicalensis, The golden herb from the garden of Chinese medicinal plants. Sci Bull. 61(2016)1391-1398

48. Kumar, S. J Van. A Review of Swertia chirayita (Gentianaceae) as a Traditional Medicinal Plant. Front Pharmacol. 6 (2015), pp. 308

49. K. Panda, L. Padhi, P. Leyssen, M. Liu, J. Neyts, and W. Luyten, Antimicrobial, anthelmintic and antiviral activity of plants traditionally used for treating infectious disease in the Similipal Biosphere Reserve, Odisha, India, Frontiers in Pharmacology., 8 (2017), pp. 658

50. Z. Moghadamtousi, H.A. Kadir, P. Hassandarvish, H. Tajik, S Abubakar, K. Zandi, A review on antibacterial, antiviral and antifungal activity of curcumin, BioMed Res Int., (2014), pp. 1-14

51. K. A.Singh, and et al., Chloroquine and hydroxychloroquine in the treatment of COVID-19 with or without diabetes: A systematic search and a narrative review with a special reference to India and other developing countries. Diabetes \& Metabolic Syndrome, Clinical Research \& Reviews., 14 (2020), pp. 241-246

52. Li, and F.Zhao, Anti-inflammatory functions of Houttuyniacordata Thunb. and its compounds: A perspective on its potential role in rheumatoid arthritis, ExpTher Med., 10 (2015), pp. 3-6

53. Salehi, N.V.A. Kumar, B. Şener, M. Sharifı-Rad, M. Kılıç, G.B. Mahady, S. Vlaisavljevic et al., Medicinal Plants Used in the Treatment of Human Immunodeficiency Virus, International Journal of Molecular Sciences, 19 (2018), pp. 1459.

54. D. Welch, K.E. Panter, D.R. Gardner, B.L. Stegelmeier, The good and the bad of poisonous plants: An introduction to the USDA-ARS Poisonous Plants Research Laboratory. Journal of Medical Toxicology; Official Journal of the American

Page 13/19 
College of Medical Toxicology., 8 (2012), pp. 153-159

55. Saha, and S. Ghosh, Tinospora cordifolia: One plant, many roles, AncSci Life, 31 (2012 ), pp.151-159

56. Kotora, F. Sersen, J. Filo, D. Loos, J. Gregan, and F. Gregan, The scavenging of DPPH, galvinoxyl and ABTS radicals by imine analogs of resveratrol, Molecules., 21(2016), pp. 127

57. Chang, K.C. Wang, C.F. Yeh, D.E. Shieh, and L.C. Chiang, Fresh ginger (Zingiber officinale) has anti-viral activity against human respiratory syncytial virus in human respiratory tract cell lines, Journal of Ethnopharmacology., 145 (2012), pp. 146-151

58. Brochot, A. Guilbot, L. Haddioui, and C. Roques, Antibacterial, antifungal, and antiviral effects of three essential oil blends, Microbiology., 6(2017)

59. Kammoun El Euch, D.B. Hassine, S. Cazaux, N. Bouzouita and J. Bouajila, Salvia officinalis essential oil: Chemical analysis and evaluation of anti-enzymatic and antioxidant bioactivities, South African Journal of Botany., 120 (2018), pp. 253-260

60. A. Aboubakr, A. Nauertz, N.T. Luong, S. Agrawal, S.A. El-Sohaimy, M.M. Youssef, et al., In Vitro Antiviral Activity of Clove and Ginger Aqueous Extracts against Feline Calici virus, a Surrogate for Human Norovirus, Journal of food protection., 79 (2016), pp. 1001-12

61. Bayan, P.H. Koulivand, A. Gorji, Garlic: A review of potential therapeutic effects, Avicenna J. Phytomed., 4(2014), pp. 114

62. M. Perez. Antiviral activity of compounds isolated from plants. Pharmaceut Biol., 41 (2003), pp.107-57

\section{Tables}

Due to technical limitations, Table 1 is only available as a download in the supplemental files section

\section{Figures}

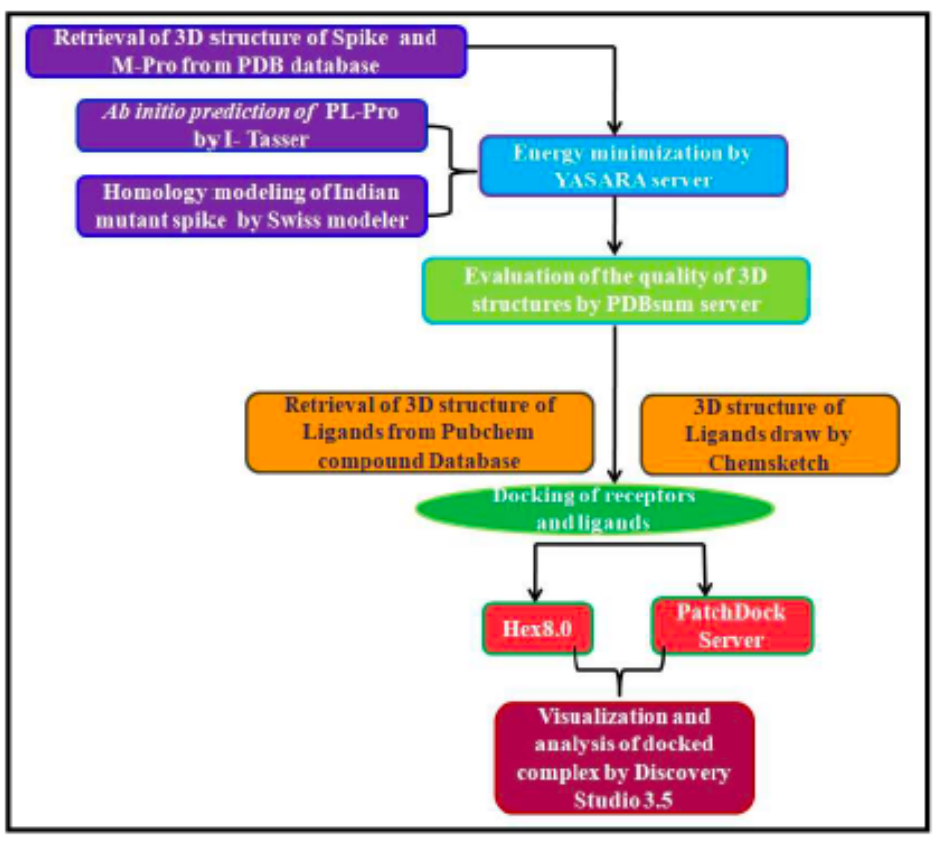

Figure 1

Experimental flow chart 


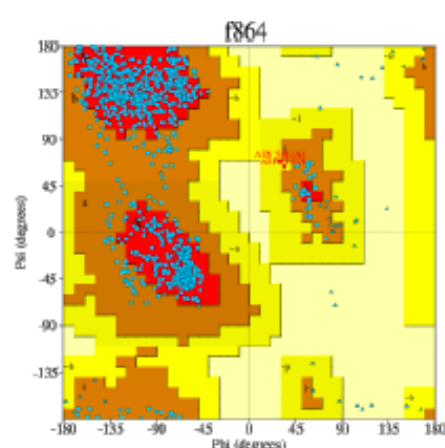

Spike

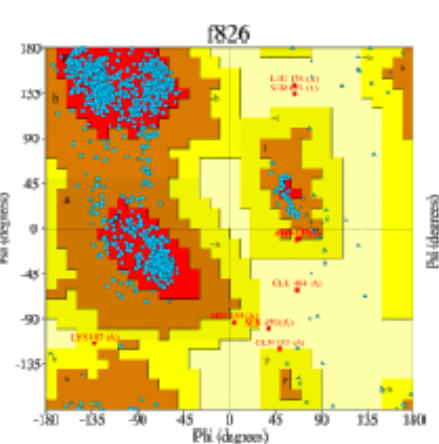

Indian mutant Spike

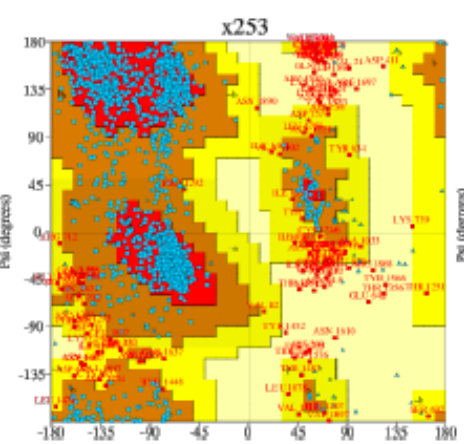

PL-Pro

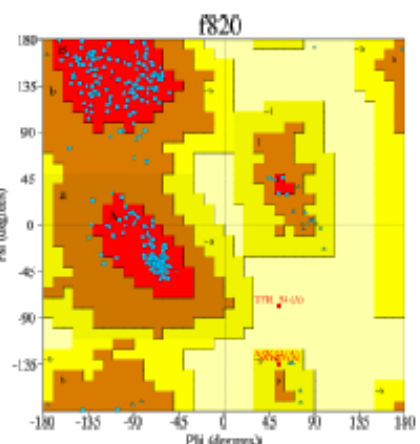

M-Pro

\begin{tabular}{|l|l|l|l|l|}
\hline COVID-19 protein & $\begin{array}{l}\text { Most favoured regions } \\
{[\mathbf{A}, \mathbf{B}, \mathbf{L}]}\end{array}$ & $\begin{array}{l}\text { Additional allowed } \\
\text { regions [a,b,l,p] }\end{array}$ & $\begin{array}{l}\text { Generously allowed } \\
\text { regions }[\mathbf{a}, \sim \mathbf{b}, \mathbf{-}, \sim \mathbf{p}]\end{array}$ & $\begin{array}{l}\text { Disallowed regions } \\
{[\mathbf{X X}]}\end{array}$ \\
\hline Spike & $83.7 \%$ & $16.0 \%$ & $0.2 \%$ & $0.0 \%$ \\
\hline IS-Spike & $86.2 \%$ & $12.8 \%$ & $0.4 \%$ & $0.5 \%$ \\
\hline PL-Pro & $74.7 \%$ & $19 \%$ & $3.8 \%$ & $2.5 \%$ \\
\hline M-Pro & $91.7 \%$ & $7.2 \%$ & $0.8 \%$ & $0.4 \%$ \\
\hline
\end{tabular}

Figure 2

Quality validation of 3D structure of Target proteins by PDB Sum
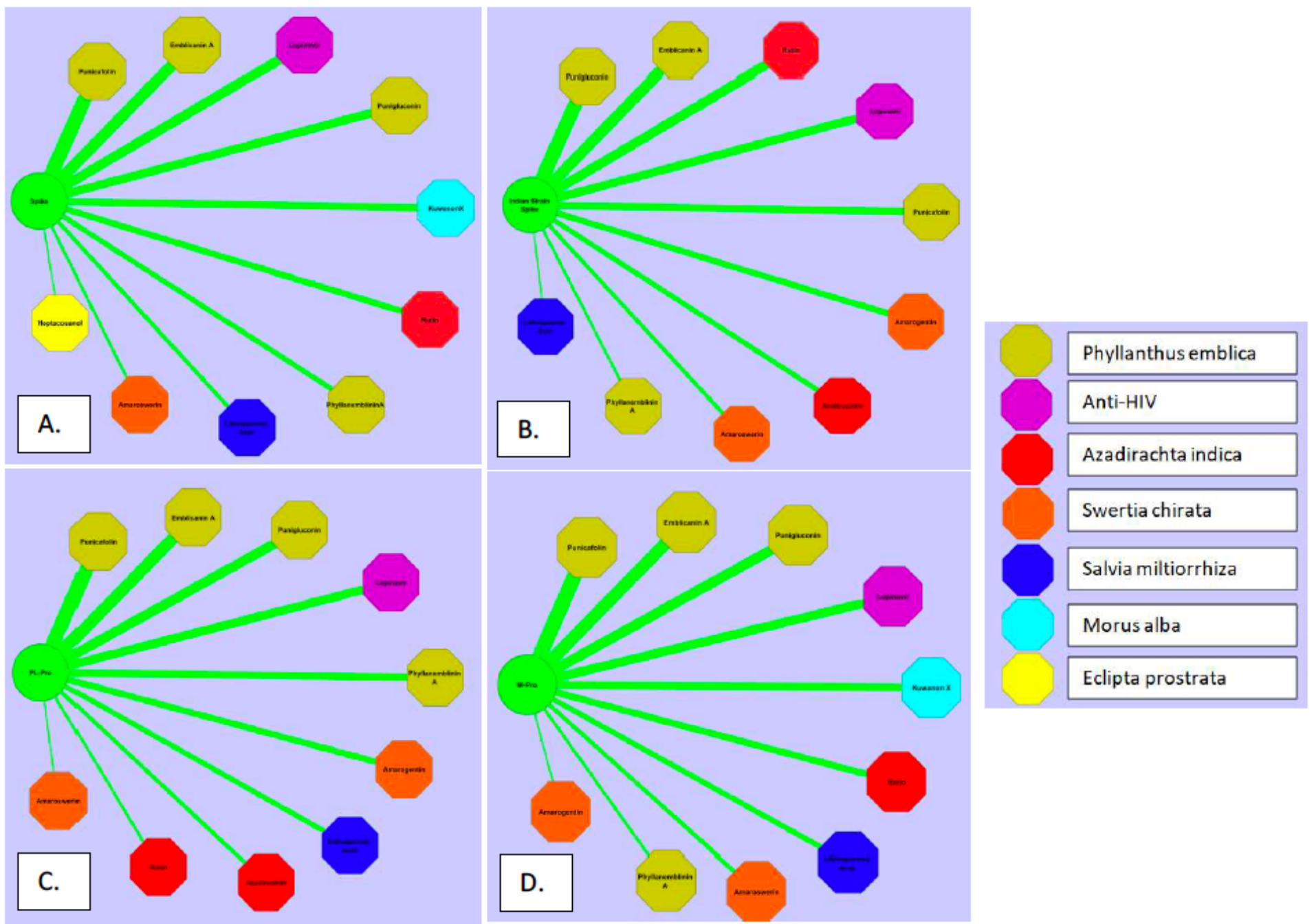


\section{Figure 3}

Network analysis of top 10 selected ligands interaction with their target proteins of COVID-19. Here green color oval shaped nodes indicate the target proteins of COVID-19 such as (A.) Spike, (B.) IS-Spike, (C.) PL-Pro and (D.) M-Pro/3CL-Pro. The octagonal shape like nodes represent ligands and their diverse color indicates different medicinal plants and a standard drug. The edge between the two nodes indicates protein-ligands interaction and has higher thickness of edge showed high binding affinity between them and vice-versa.
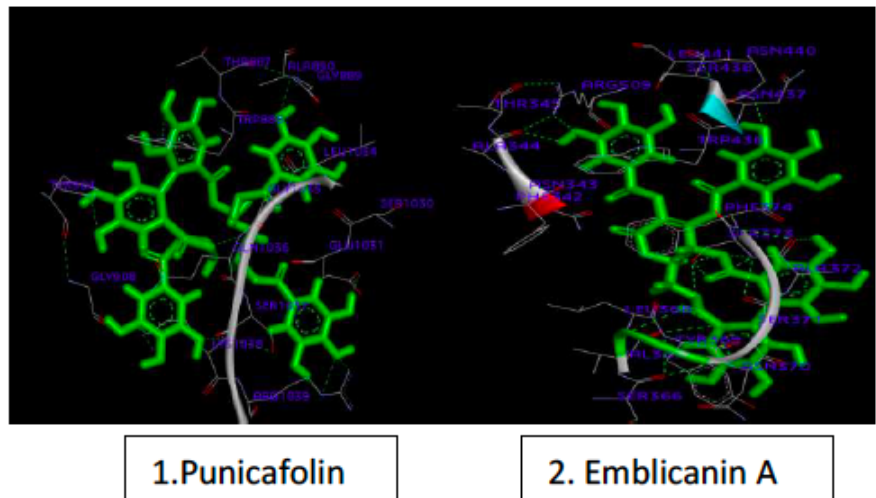

\section{Emblicanin A}

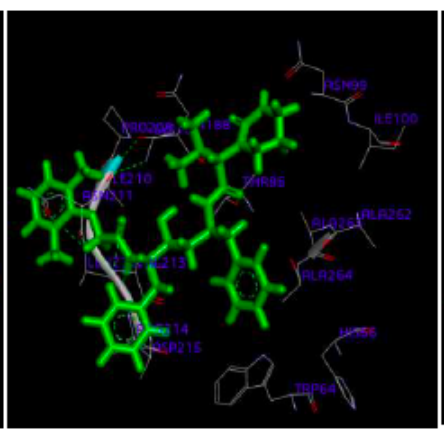

3. Lopinavir

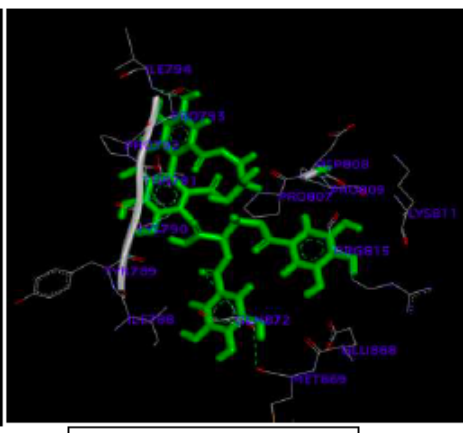

4. Punigluconin

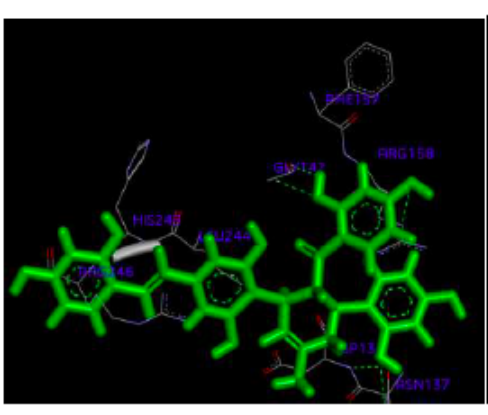

5. Kuwanon X

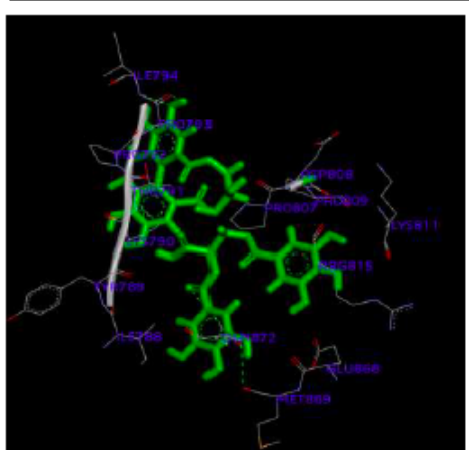

9. Amarosweri

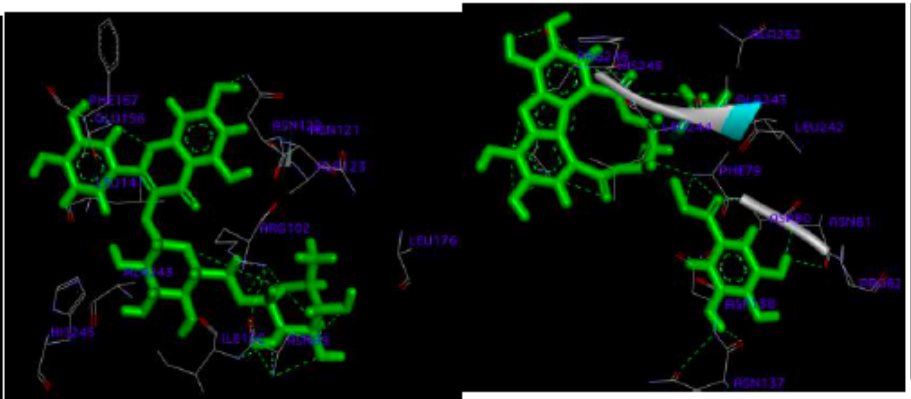

7. Phyllanemblinin Acid

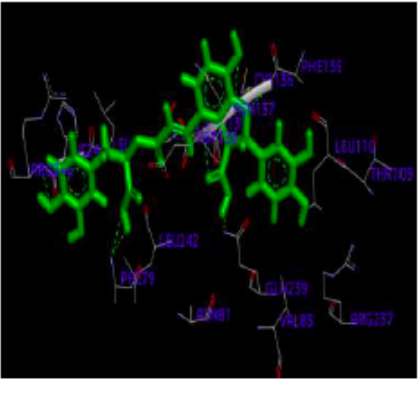

8. Lithospermic Acid

\section{Figure 4}

Experimentally determined 3D plot of top 10 leading Spike-ligands interaction. Green colour dash indicates H-bond interaction between atoms. 


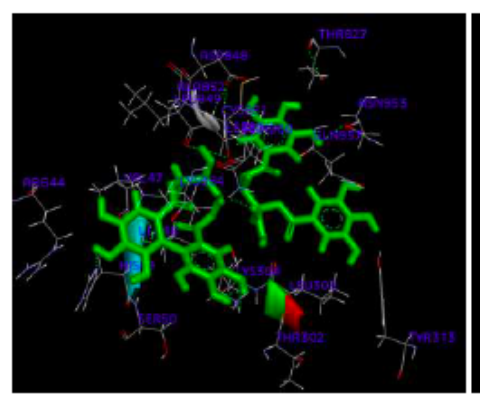

1. Punigluconin

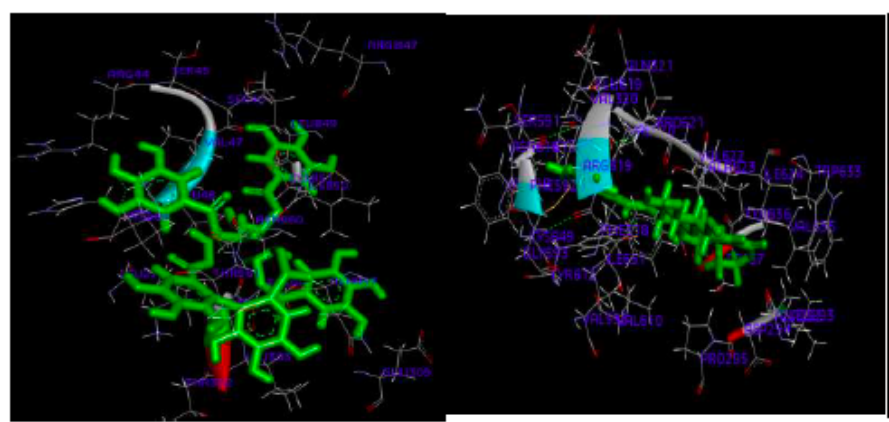

5. Punicofolin

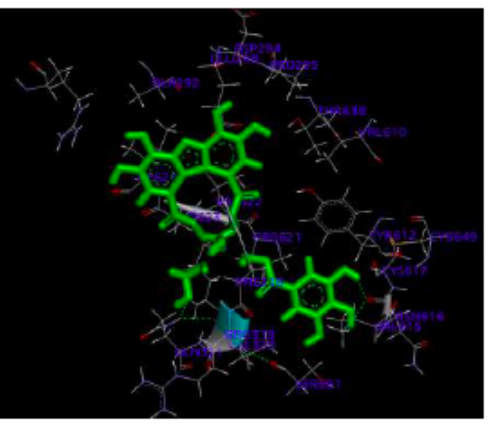

9. Phyllanemblinin

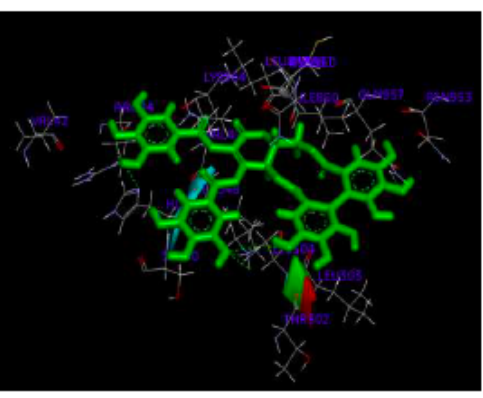

2. Emblicanin A

6. Amarogentin

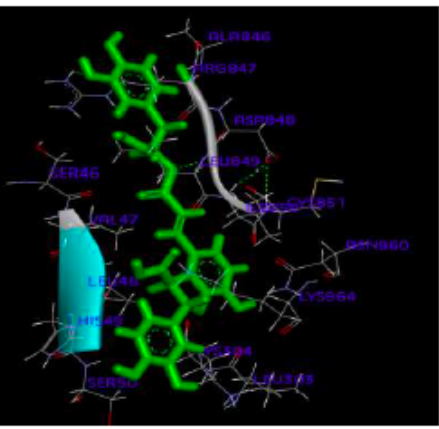

10. Lithospermic Acid

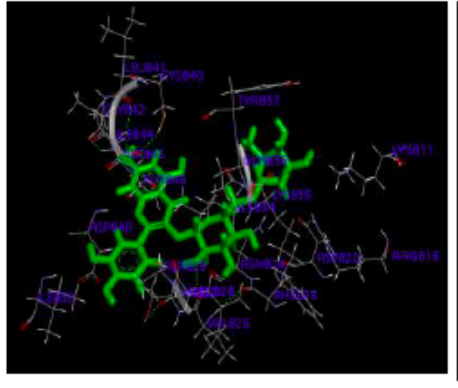

3. Rutin

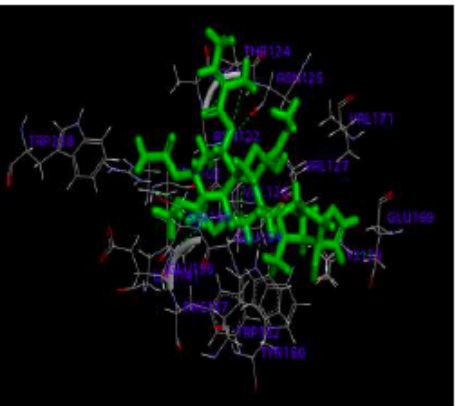

7. Azadirachtin

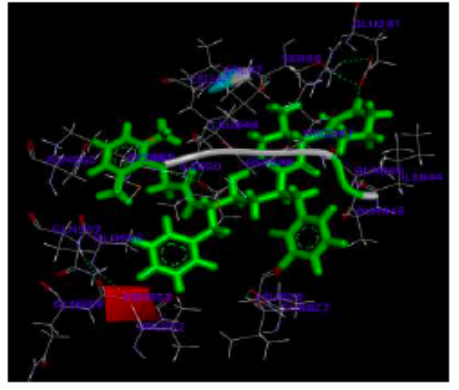

4. Lopinavir

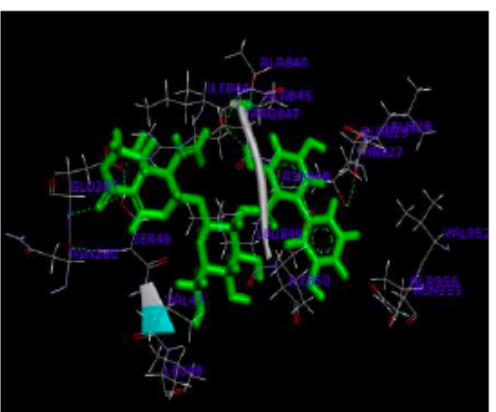

8. Amaroswerin

\section{Figure 5}

Experimentally determined 3D plot of top 10 Indian strain Spike-ligands interaction. Green colour dash indicates $\mathrm{H}$-bond interaction between atoms. 


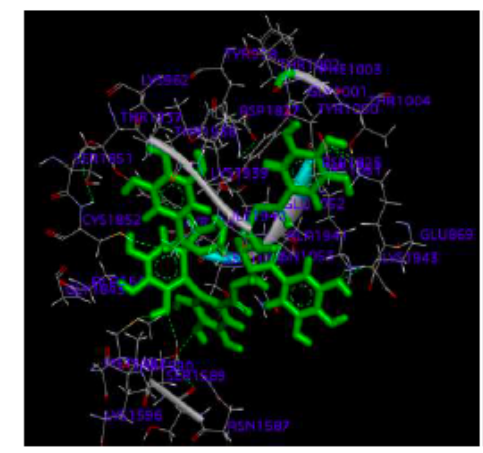

1.Punicofolin

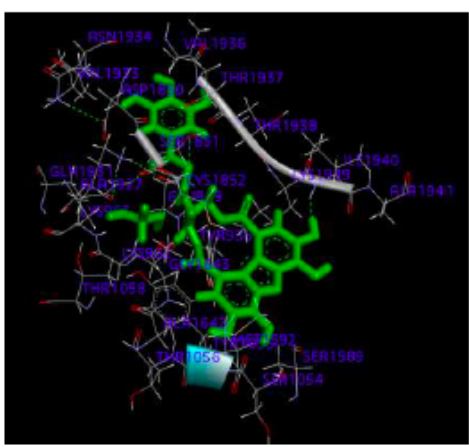

5. Phyllanemblinin A

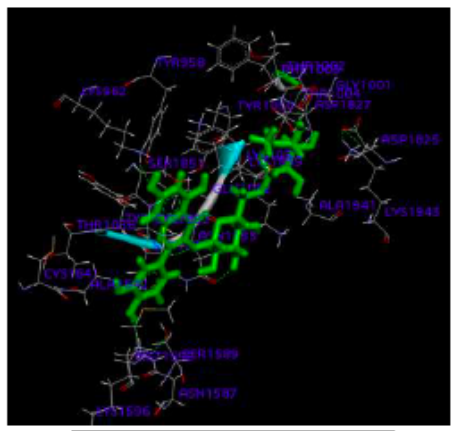

9. Rutin

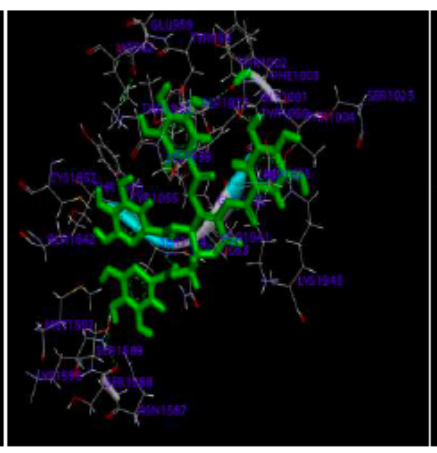

2. Emblicanin A

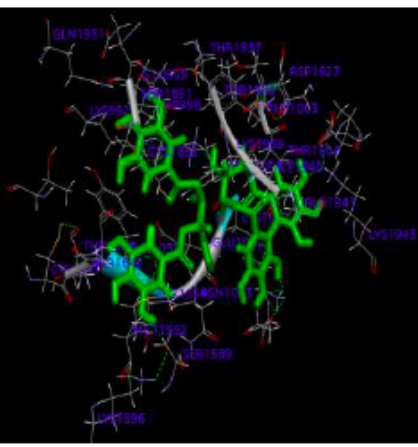

3. Punigluconin

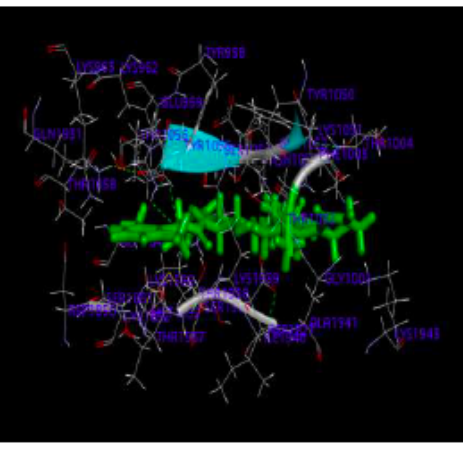

4. Lopinavir

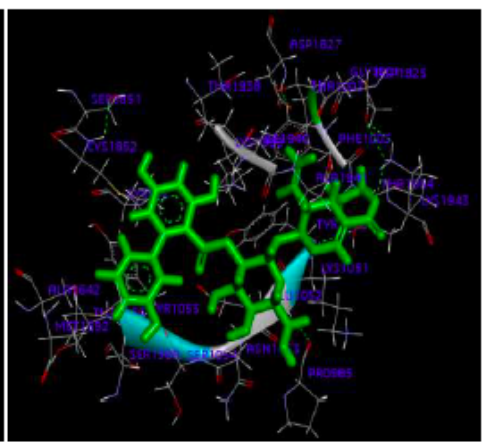

6. Amarogentin

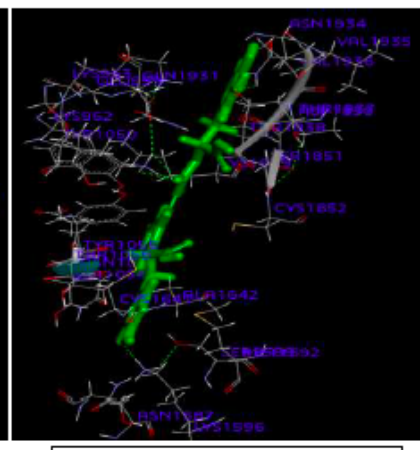

7. Lithospermic Acid

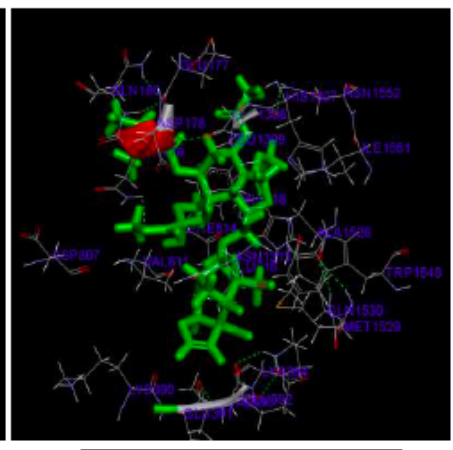

8. Azadirachtin

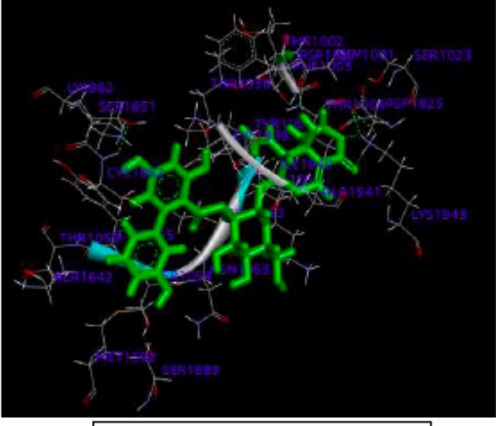

10. Amaroswerin

Figure 6

Experimentally determined 3D plot of first 10 PL-Pro -ligands interaction. Green colour dash indicates H-bond interaction between atoms. 


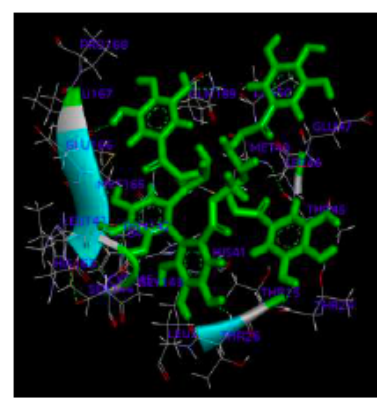

1.Punicafolin

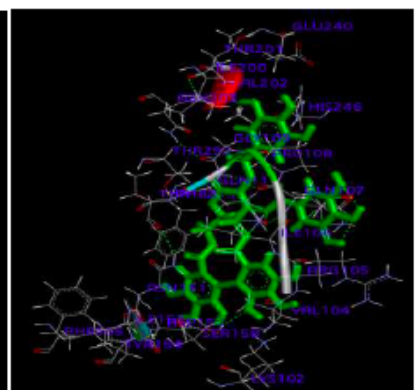

2. Emblicanin A

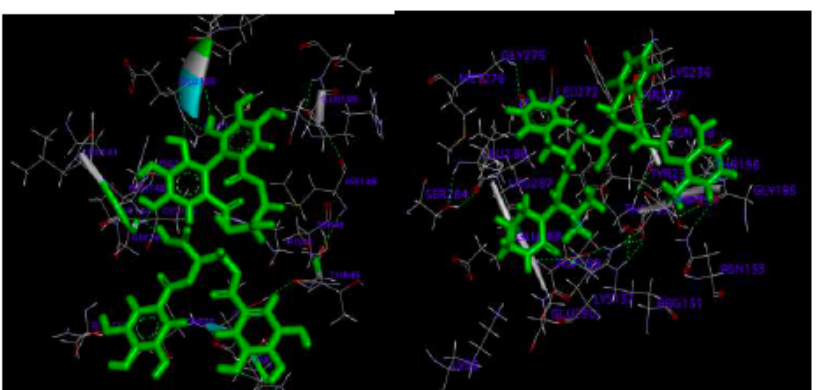

3. Punigluconin

4. Lopinavir

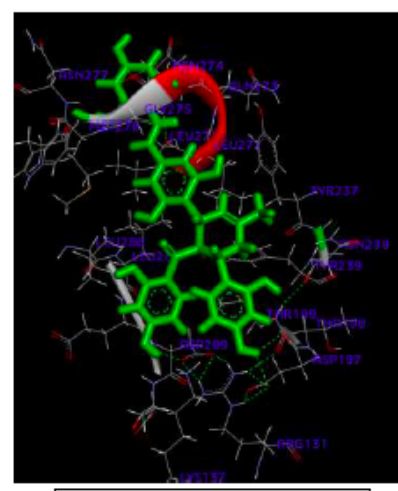

5. Kuwanon X

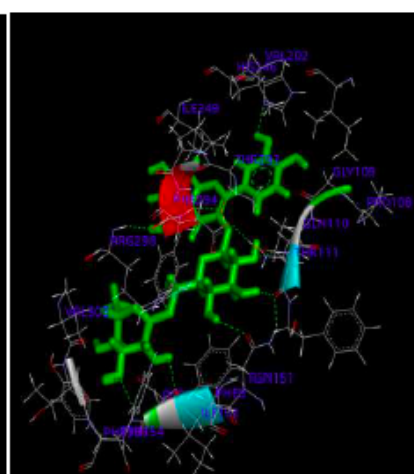

6. Rutin

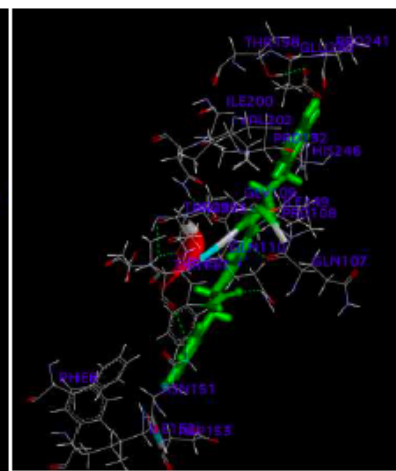

7. Lithospermic Acid
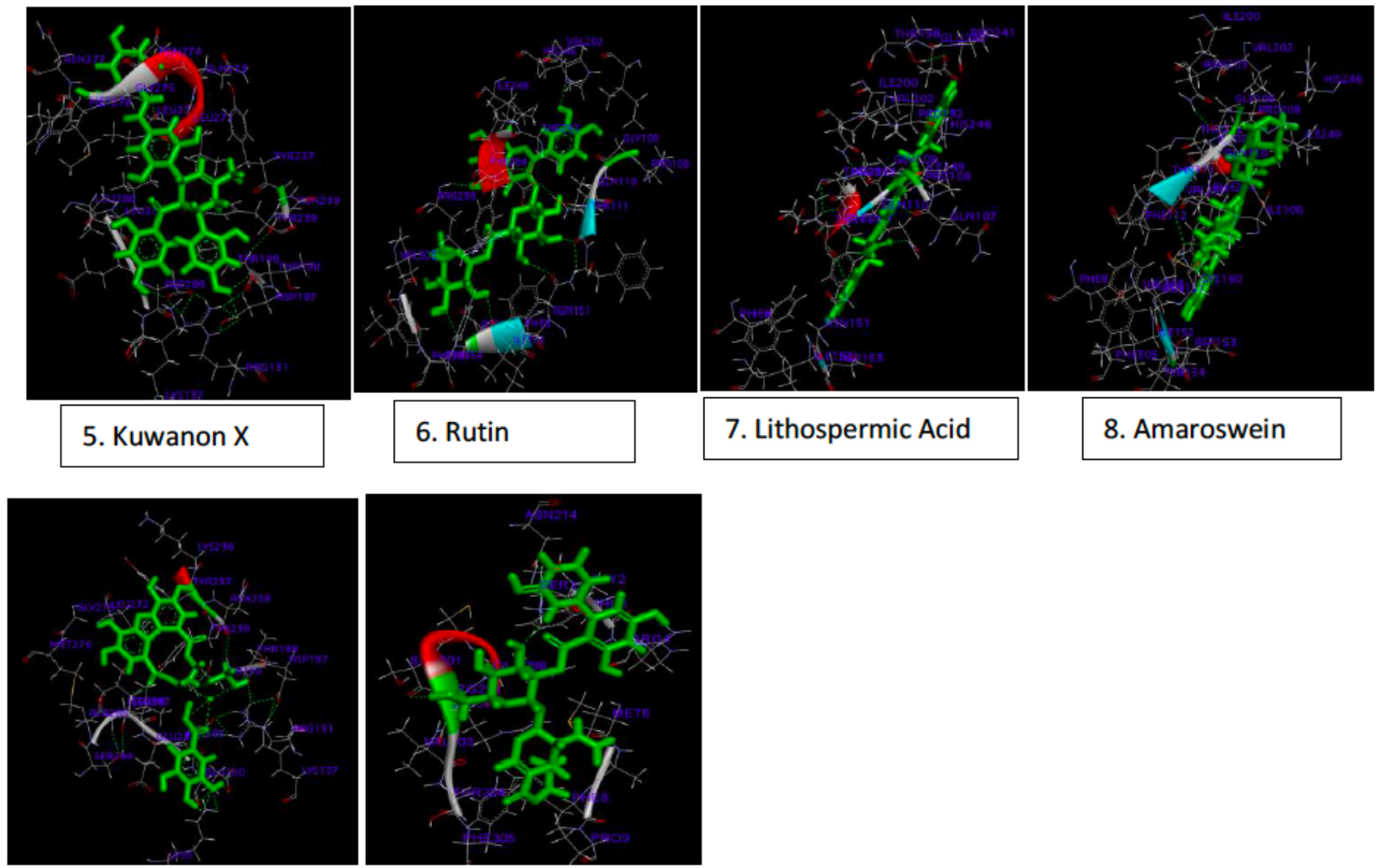

9. Phyllanemblinin A

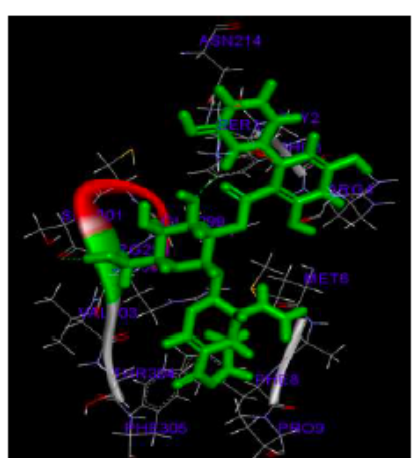

10. Amarogentin

Figure 7

Experimentally determined 3D plot of ten main M-Pro-ligands interaction. Green colour dash indicates H-bond interaction between atoms.

\section{Supplementary Files}

This is a list of supplementary files associated with this preprint. Click to download.

- Table1.docx 